\title{
The need for an Inclusive Space Sector: a Student Perspective
}

\author{
A. C. O'Brien, L. J. Martin and S. Lee Roberts \\ UK Students for the Exploration and Development of Space \\ aine.obrien@ukseds.org
}

\begin{abstract}
The UK Space Agency aims for the sector to account for $10 \%$ of the global sector by 2030 [1]. This target cannot be achieved without growing the workforce to support the sector, which will require removing barriers to accessibility for underrepresented minorities, making the sector inclusive for all. UKSEDS is working to ensure that its own practices are inclusive; this paper presents UKSEDS inclusivity initiatives and demographic data, including ethnicity, gender and sexual orientation. Comparisons are made to workforces from the space and other sectors, and overall, delegates at the National Student Space Conference (NSSC) are more diverse.
\end{abstract}

Keywords-diversity, inclusion, space sector, students, equality

\section{INTRODUCTION}

UK Students for the Exploration and Development of Space (UKSEDS) is the UK's national student space society. The society was created in 1989 and seeks to create opportunities for students and graduates interested in space. It has almost 500 members, comprising primarily of physics and engineering university students.

A. Aims

This paper seeks to:

1. Outline the motivations for working towards a diverse and inclusive space sector;

2. Summarise the demographics of the students taking part in recent UKSEDS activities, and compare this to the sector as a whole;

3. Explain the work UKSEDS is doing to make space accessible to all;

4. Set out future plans in this area for UKSEDS

This paper serves as an update to a poster presented at the Lunar and Planetary Science Conference in Texas, 2019 [2].

\section{A. Introduction to UKSEDS}

UKSEDS is run nationally by a team of $\sim 30$ volunteers, remotely, across the UK. There are also currently 27 branches; these are space-related university societies which have affiliated with UKSEDS in the 2018/19 academic year. The main activities UKSEDS organises are outlined below:

Events and Workshops - conferences and training to inform, enthuse and upskill students in the space sector, including:
- National Student Space Conference (NSSC) is UKSEDS' largest annual two-day event which sees $\sim 350$ students attending, its location changes annually.

- Careers Launch, these events are smaller scale one-day careers conferences held for students and school pupils.

- Diversity in Space Careers (DISC), one-day conference, held at the Royal Astronomical Society in 2018, to promote equality, diversity, and inclusion in the space sector.

Competitions - Technical team project-based challenges for university students to gain problem solving experience in space related disciplines, including:

- Rover competition, each year since 2016, student groups have been invited to design, build, and test a rover. The competition spans a year, with each design phase judged by industry professionals, and a final testing day at Rutherford Appleton Laboratory (RAL) Space facility.

- National Rocketry Championships, a summer competition for student groups to build, launch and learn about hobbyist rocketry.

Advocacy - Voicing the needs of students and those in their early career in the space sector to key stakeholders, some activities carried out include:

- Speaking/participating in panels at external events.

- Writing papers outlining the student perspective on key issues for the sector.

Outreach - Volunteers taking part in science communication and public engagement.

Careers - Running the SpaceCareers.uk website, a careers resource for young people, students and graduates interested in working in the space sector.

\section{B. The UK Space Sector at a Glance}

In $2016 / 17$ the space sector in the UK was worth $£ 14.8$ bn, accounting for $5.1 \%$ of the global space economy [1]. The UK Space Agency aim for the sector to make up $10 \%$ of the global sector by 2030; this means that a much larger workforce is needed [1]

UKSEDS considers the space sector to include scientific and technological research, academia, non-technical areas (such as outreach/public engagement and space law) and industry. The 
space industry is split into two segments, upstream and downstream. Upstream involves activities related to the manufacture and launch of satellites and spacecraft, including the research and development stages, and any associated products, services and infrastructure. The downstream segment utilises the space technologies and data from space assets to deliver products and services such as satellite broadcasting and Earth observation. In 2016/17 the downstream segment accounted for the majority of the UK space industry's income at $£ 12.4$ bn, while upstream increased from previous years to $£ 2.4$ bn [1].

II. Motivations FOR Diversity AND INCLUSION WORK 'Diversity and inclusion' is the advocation of the involvement and empowerment of individuals with different genders, sexual orientations, ethnicities and socio-economic backgrounds. This goes beyond a basic tolerance of people's differences, by aiming to understand, respect, and accept one another. Within a workforce, this can even result in the development of specific strategies and practices to support the growth of diverse groups. Here we use the term 'diversity' to mean the representation of different groups within a field or organisation, whilst 'inclusion' means the behaviours and actions that promote equality and diversity.

This is not just an issue within the space sector, but is being addressed globally by governments and other organisational bodies. In the past, United Nations agencies and others have run campaigns including "Do Something For Diversity and Inclusion" and "World Day for Cultural Diversity for Dialogue and Development" [3], in order to raise awareness around such issues. These activities lie under the UN's Sustainable Development Goals which include gender parity. The UK government has also passed several laws promoting diversity and inclusion, including the Equality Act 2010 [4] and have put in place systems to monitor metrics, such as the gender pay gap, by which companies with over 250 employees must report the disparity in wages between different genders within the company.

Across all areas of STEM, initiatives have been implemented to measure diversity within the workforce, and to advocate for a more inclusive field. For example, the Royal Society collects demographic data and found only $9 \%$ of fellows identified as female in 2018 [5]. Other charities and initiatives such as the WISE Campaign and Stemettes have been set up to address gender imbalance in STEM. The Royal Academy of Engineering established the Engineering Diversity Concordat as a means for professional engineering institutions to commit to increasing diversity. There is a need for such work as just $7.8 \%$ of engineering professionals in 2018 were from BAME (black, Asian and minority ethnic) backgrounds, this is less than the $12 \%$ for the total UK workforce [6].

Within the space sector in the UK, professional bodies and learned societies are also promoting diversity and inclusion efforts. The Royal Astronomical Society (RAS) 2016 demographics survey of the Astronomy and Geophysics research community [7] found that minorities, such as those who identify as BAME and disabled people are under-represented at all levels in the field. The Women in Aviation and Aerospace Charter was developed as a commitment to improving the gender balance in aviation and aerospace, particularly supporting women into levels of seniority [8]. Several organisations support the charter including the Royal Aeronautical Society and Women in Aerospace Europe - UK, and signatories include the UK Space Agency.

The RAS alongside the Institute of Physics and the Royal Society of Chemistry published a report in 2019 which surveyed physical scientists in the UK and Ireland to investigate the climate for LGBT+ people in the workplace. $28 \%$ of the LGBT+ respondents to this survey said that they have at some stage considered leaving their job as a result of LGBT+-related discrimination in the workplace [9]. The report made recommendations for improving the climate for LGBT+ people, for example: improved training which can tackle issues such as misgendering.

Diversity and inclusion in the workplace is not only important from a moral perspective, but could also have economic benefits. While data suggest that more diverse organisations are more likely to perform better [10] it is difficult to prove that this enhanced performance is down to increased diversity, as companies with more diverse workforces are more likely to be more forward thinking, on the whole [11]. This should not, however, deter organisations from working towards a diverse workforce, since it is clear that if barriers exist for some groups of people then the talent pool being drawn from is reduced.

Since early 2018, UKSEDS has focused many of its activities on advocating for a diverse and inclusive space sector in the UK and Europe. The space sector in the UK is less diverse than the UK workforce. People of colour, women, people with disabilities, LGBTQ+ people and those from low socioeconomic backgrounds are under-represented, especially in more senior roles, both in STEM, and in the space sector. There is already a shortage of skilled workers in STEM in the UK [1]. In order to reach the expansion goal for the sector, it is clear the space industry needs to diversify.

UKSEDS believes that everyone, regardless of background, should have equal access to the space sector, and is therefore working to advocate for a more inclusive field. Most organisations focus on diversity and inclusion work with school pupils or those working within their own companies. UKSEDS acts as a gateway to the sector for many students and is championing best practice in inclusivity and reflection on its own activities, to ensure that we make space accessible to all, to make the sector inclusive and welcoming for the incoming workforce.

UKSEDS has been working to determine the demographics of its membership, by surveying participants of each activity. The objective is to identify any differences in the backgrounds of students taking part in the various categories of our events and competitions, identify reasons for this, and find out how better to support diversity in these settings. These will be monitored over the coming years, to build a picture of the demographics, and to identify any trends. 


\section{Diversity DATA From UKSEDS ACtIVITIES}

\section{A. Methods}

An optional diversity survey is part of the online registration process for anyone participating in any UKSEDS event or competition. The survey asks respondents for their gender, sexual orientation and ethnicity.

Over time, UKSEDS intends to build up a database of information about the diversity of our audience, how that is changing and to try to identify possible reasons for this.

This paper represents the first set of data that has been collected since the start of this initiative. Data from NSSC 2019, DISC 2018 and the 2018 \& 2019 rover competition are displayed, alongside reference datasets.

\section{B. Results}

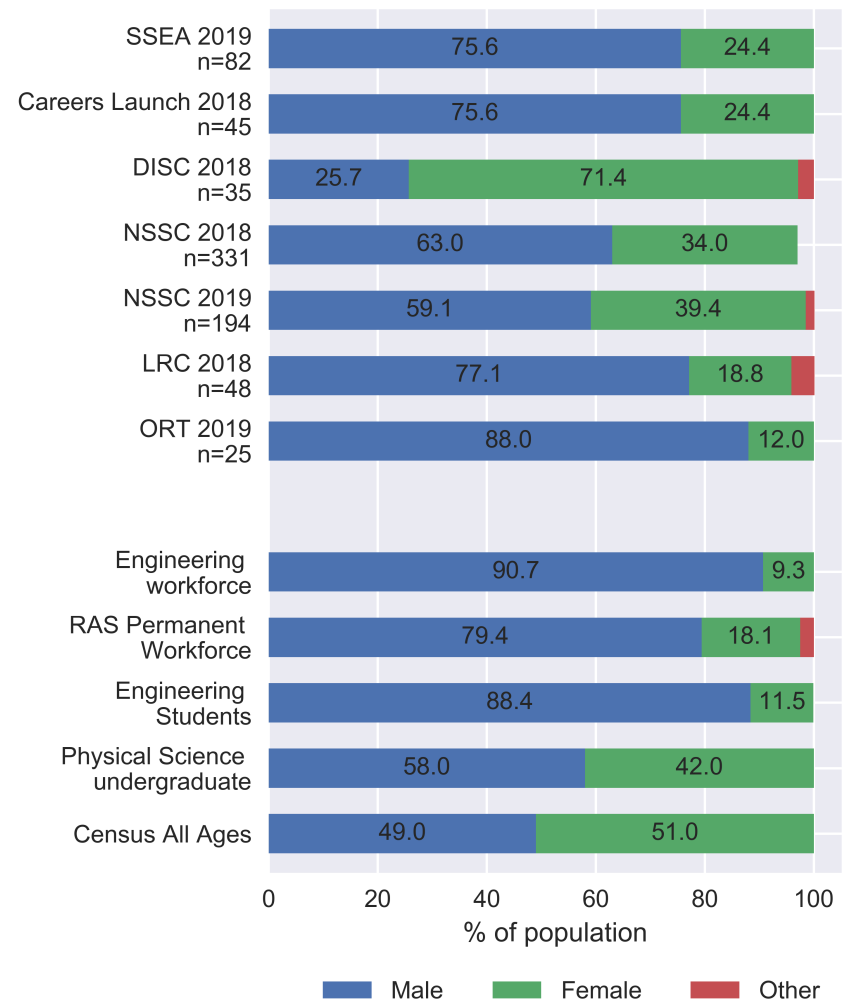

Fig. 1 The genders of people who attended UKSEDS events in 2018/19 are displayed, alongside reference datasets from refs $[6,7,12,13]$.

\section{Discussion}

Comparing Figures 1-3, we can see that NSSC delegates are more diverse, on the whole, than their space-related workforce counterparts. The engineering and astronomy workforces, for example, have a much lower proportion of women than NSSC had in 2019 . Similarly, only $81.7 \%$ of NSSC survey respondents identified as white, compared to $90 \%$ of the astronomy workforce.

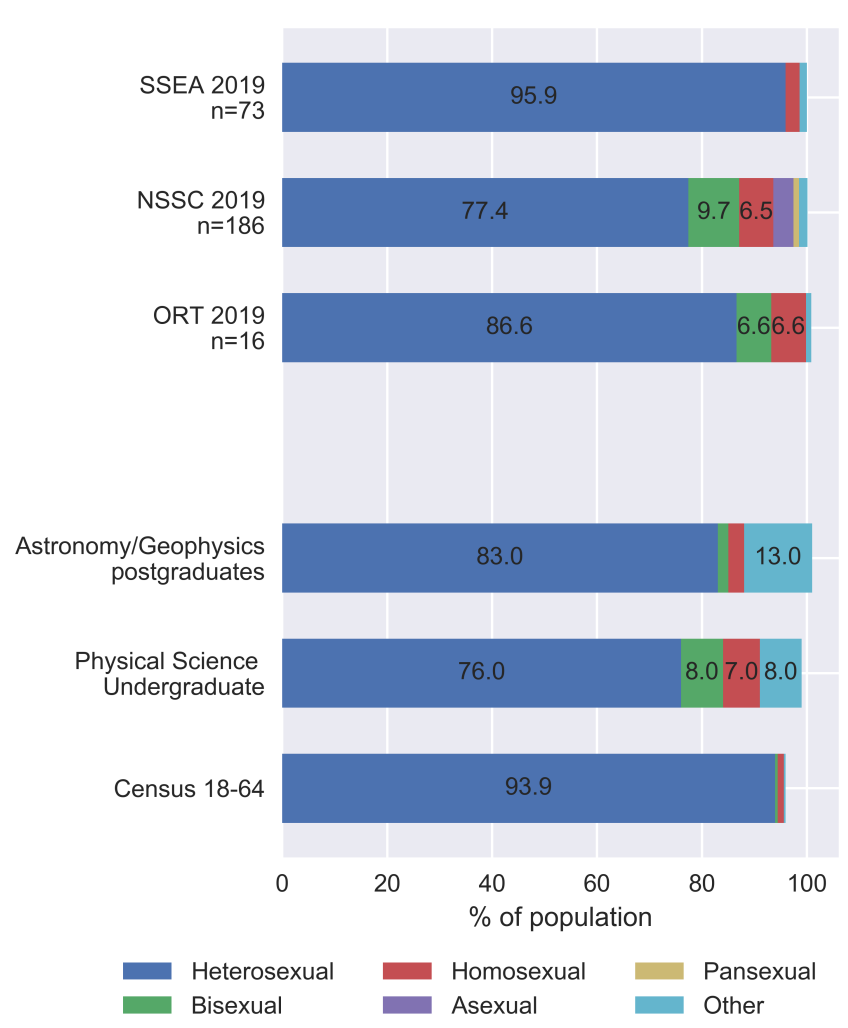

Fig. 2 The sexual orientation of delegates at UKSEDS events in 2018/19 are displayed, alongside relevant available reference datasets from refs $[6,7,12,13]$.

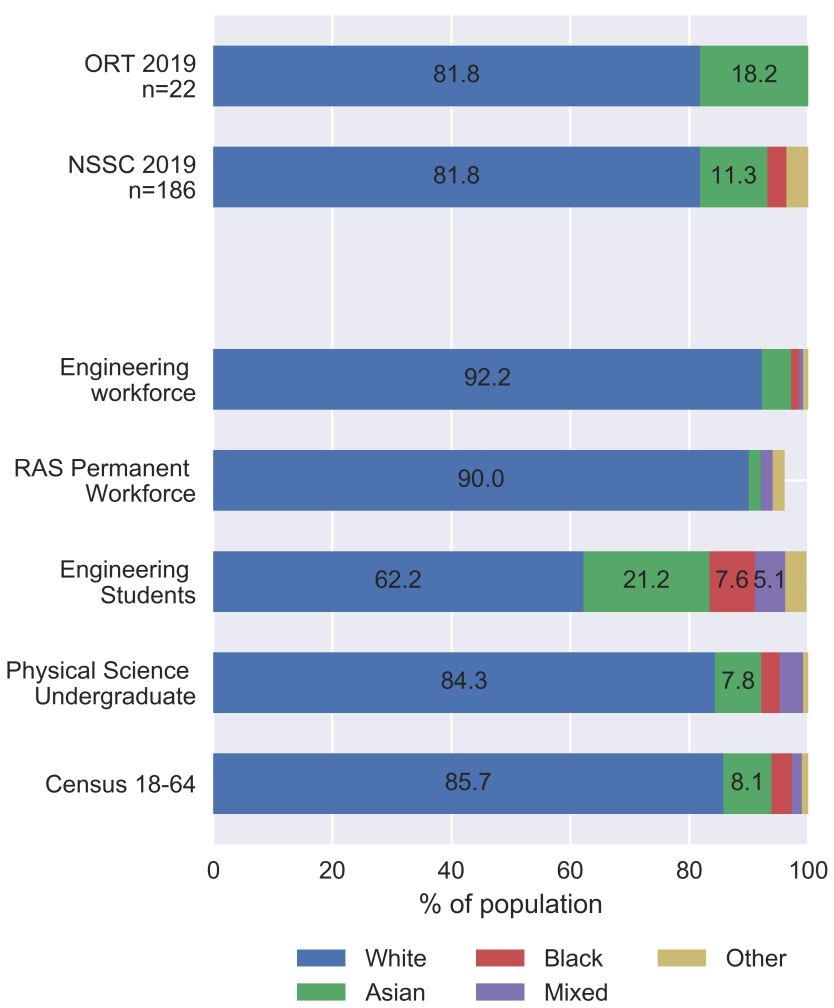

Fig. 3 The ethnicities of delegates at two UKSEDS events are displayed, alongside relevant available reference datasets from refs $[6,7,12,13]$. 
NSSC is open to all, however most attendees $(\sim 70 \%)$ are either engineering or physics students. Comparing these datasets presents difficulties and limitations when making inferences, due to the differences in surveys by external organisations. The figures shown for physical science undergraduates are from the Higher Education Statistics Authority (HESA) which collects data for all physical sciences, as a whole, rather than individual subject areas, such as physics or chemistry. This, in particular, is likely to skew the data from a gender perspective, since $\sim 50 \%$ of A level chemistry pupils are girls, compared to only $\sim 20 \%$ of A level physics pupils. It is therefore likely that, if we were to compare our data to physics undergraduates, as opposed to physical science undergraduates, the NSSC delegates would have a significantly higher proportion of women. Should this assumption be correct, it may well be the case that more women are interested in space than are interested in other areas of physics.

It is clear from Figure 1 that there is a difference in gender participation in different categories of UKSEDS events. A much higher proportion of the delegates at DISC 2018, for example, were women, compared to the participants in the Lunar Rover Competition (LRC) and Olympus Rover Trials (ORT) finals days in 2018 and 2019. DISC was a one-day conference held to champion equality, diversity, and inclusion in the space sector, whilst the rover competitions' finals days consisted of talks and technical testing of small planetary rovers. Most competitors in the rover competition are engineering students; from Figure 1, we can see that the proportion of women participating in the rover trials finals is broadly representative of the undergraduate cohort.

Looking at Figure 1, many UKSEDS events have a similar gender ratio in attendance as this symposium, however our larger scale NSSC events, as well as DISC, both have a larger proportion of women than this symposium, the third symposium for space educational activities (SSEA) in attendance. It is difficult to discuss race and ethnicity at SSEA, as there is variation across Europe as to the definitions of race vs ethnicity vs nationality. Since ethnicity pertains to shared cultural heritage, there is wide variation amongst European countries as to what counts as an ethnic group. Thus, a direct comparison can therefore not easily be made between SSEA data and other UKSEDS activities, regarding ethnicity; the authors believe a more appropriate question would have been to ask delegates their nationality and indeed UKSEDS should start asking for nationality, too, in the future. The largest issue with the dataset that has been collected to date is the limited sample size. UKSEDS has a registered membership of around 500 members, as well as event and competition attendance of around 500, which is a limited pool of people, especially when trying to understand the effects of various influences (such as inclusivity initiatives) on diversity. Additionally, reference data for the diversity of the space sector's workforce is limited, particularly on the industrial side.

\section{INCLUSIVE INITIATIVES}

In order to make space accessible to all, UKSEDS has been working to make its events and activities as inclusive as possible. A few of its initiatives are outlined below, along with the associated rationale.

\section{A. Gender Pronoun Stickers}

At all UKSEDS events, all attendees (including speakers and sponsors) are asked to declare their gender pronouns (e.g. she/her/hers) by writing them on a sticker, see Figure 4 . This simple activity reduces the risk of a delegate misgendering another delegate, without having to ask them for their pronouns directly. Asking all attendees to use gender pronoun stickers/badges also normalises the act, so that assumptions never need to be made and the right to self-identify is respected.

\section{B. Colour Communication Stickers}

UKSEDS asks all its delegates to use a traffic light system to quickly and clearly display their preferences whilst in networking breaks. Conferences can be challenging environments, and some people may have difficulty initiating conversations with others, whilst other people may not wish to converse with others at all. This scheme is adapted from [14] and the colour code is outlined below:

- Red: someone who does not wish to be spoken to by anyone whilst this sticker is on display, they may, however, choose to approach people;

- Orange: someone who only wishes to be approached by people they know, they may, however, choose to approach other people;

- Green: someone who is happy to be approached by anyone, they may also find it difficult to approach others themselves.

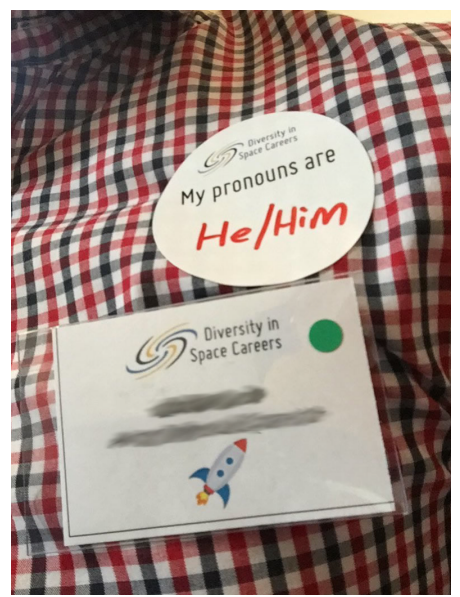

Fig. 4 The gender pronoun and colour communication stickers used by UKSEDS at its events.

UKSEDS is determined to work on diversity and inclusion with an intersectional approach, and recognises that whilst this traffic light system is effective for most people to understand others' communication preferences, people who are red/green colourblind may struggle to distinguish the difference. UKSEDS is therefore going to improve these stickers through the use of patterns/graphics, to make these stickers more inclusive.

\section{Diverse Speakers and Chairs}

UKSEDS recognizes the importance of diverse role models and ensures that speaker lists and panel discussions at all its events are as diverse as possible. For NSSC, an equal 
female:male ratio has been maintained alongside a wide range of other backgrounds, such as BAME and LGBTQ+.

In order to encourage and build confidence in students/those in their early career, UKSEDS has incorporated a chair and cochair system for panel discussions, whereby a less experienced person chairs the discussion alongside a person with more experience. Having a chair and co-chair system also encourages a wider range of questions to be asked.

\section{Anonymous Question Submission}

At NSSC, audiences are given the option to submit questions to speakers and panelists anonymously, whereby an online form specific to the session they are in. The session chairs can access these questions and put them to the speakers, giving the opportunity for questions to be asked by people who feel less comfortable asking them publicly.

\section{E. Breakout Zones}

At its larger events UKSEDS designates a room for delegates to use as a quiet space, to have a break from networking. This room is well sign-posted and delegates are able to use it at any time during the conference.

\section{F. Code of Conduct and Reporting}

UKSEDS has a clear code of conduct for delegates and volunteers at its events, to set out expectations for behaviour, to maintain a safe, enjoyable and accessible environment for all.

\section{FUTURE Plans}

As previously mentioned, we intend to continue surveying diversity at our own events over the next few years, to build a clearer picture of how we are facilitating inclusion, as an organisation. We will also solicit feedback on our own inclusivity initiatives, to see how they are being received, and if we can improve our approach to these situations.

In addition to this, we would like to engage further with other organisations about how they collect their own data and promote diversity and inclusion, both within their workplaces and at public events.

\section{CONCLUSIONS}

As the main representing body for students interested in entering the space sector, UKSEDS acknowledges its duty to set an example of a diverse volunteer workforce and inclusion throughout all of its activities. Through this ongoing work advocating for a more inclusive sector, we have identified practice that would be useful for colleagues and stakeholders. We will continue to use and refine these, to promote diversity in the best way possible, and encourage others in the sector to do the same.
UKSEDS also strongly encourages like-minded organisations, societies and businesses to collect anonymous data on the diversity of their volunteers, staff and members and to make this available to others. This will build a wider pool of data and allow the sector to identify any barriers to the sector with more accuracy, and thus create a more targeted approach to removing them.

\section{REFERENCES}

[1] UK Space Agency, "The size and health of the UK space industry 2018" 2018, available from: https://www.gov.uk/government/publications/ukspace-industry-size-and-health-report-2018 [accessed 01/08/2019]

[2] A. C. O'Brien et al., "Diversity in student space activities in the United Kingdom", 2019, 50 ${ }^{\text {th }}$ Lunar and Planetary Science Conference, The Woodlands, Texas, (Abstr.) available from https://www.hou.usra.edu/meetings/lpsc2019/pdf/2380.pdf

[3] United Nations "Do One Thing for Diversity and Inclusion" https:/www.unaoc.org/what-we-do/campaigns/do-one-thing-fordiversity-and-inclusion/ [accessed 15/07/2019]

[4] UK Government Department for International Development "Our equality and diversity strategy" available from https://www.gov.uk/government/organisations/department-forinternational-development/about/equality-and-diversity [accessed 20/07/2019]

[5] The Royal Society, "Diversity data report 2018" 2019, available from https://royalsociety.org/-/media/policy/topics/diversity-inscience/Annual-diversity-data-report-2018.pdf [accessed 05/8/2019]

[6] S. Neave et al., "Engineering UK: The state of engineering", 2018 available from https://www.engineeringuk.com/media/156187/state-ofengineering-report-2018.pdf [accessed 08/08/2019]

[7] Royal Astronomical Society "The demographics and research interests of the UK astronomy and geophysics communities 2016", 2017, available from https://ras.ac.uk/ras-policy/communitydemographics/demographic-survey-2017 [accessed 10/03/2019]

[8] UK Government "Women in aviation and aerospace Charter", 2019, available https://assets.publishing.service.gov.uk/government/uploads/system/uplo ads/attachment_data/file/797195/Women_in_Aviation_and_Aerospace_ Charter_24_April_2019.pdf [accessed 01/08/2019]

[9] Royal Society of Chemistry, Institute of Physics and Royal Astronomical Society "Exploring the workplace for LGBT+ physical scientists", 2019 available from https:/www.rsc.org/campaigningoutreach/campaigning/incldiv/lgbt-report/ [accessed 01/08/2019]

[10] McKinsey and Company "Why diversity matters", 2015, available from https://www.mckinsey.com/business-functions/organization/ourinsights/why-diversity-matters [accessed 08/08/2019]

[11] Chartered Institute of Personnel and Development "Diversity and inclusion at work: facing up to the business case" https://www.cipd.co.uk/knowledge/fundamentals/relations/diversity/dive rsity-inclusion-report [accessed 08/08/2019]

[12] Higher Education Statistics Agency "Who's in HE?" available from https://www.hesa.ac.uk/data-and-analysis/students/whos-in-he [accessed 01/02/2019]

[13] UK Government Office for National Statistics "2011 Census"

[14] Autistic Self Advocacy Network "Color communication badges" available from https://autisticadvocacy.org/wpcontent/uploads/2014/02/ColorCommunicationBadges.pdf [accessed 01/09/2018] 